University of Wollongong

Research Online

Faculty of Science, Medicine and Health -

Papers: Part B

Faculty of Science, Medicine and Health

$1-1-2019$

\title{
Dasymaschalolactams A-E, Aristolactams from a Twig Extract of Dasymaschalon dasymaschalum
}

\author{
Virayu Suthiphasilp \\ Mae Fah Luang University \\ Wisanu Maneerat \\ Mae Fah Luang University \\ Raymond J. Andersen \\ University of British Columbia \\ Piyaporn Phukhatmuen \\ Mae Fah Luang University \\ Stephen G. Pyne \\ University of Wollongong, spyne@uow.edu.au
}

See next page for additional authors

Follow this and additional works at: https://ro.uow.edu.au/smhpapers1

\section{Publication Details Citation}

Suthiphasilp, V., Maneerat, W., Andersen, R. J., Phukhatmuen, P., Pyne, S. G., \& Laphookhieo, S. (2019). Dasymaschalolactams A-E, Aristolactams from a Twig Extract of Dasymaschalon dasymaschalum. Faculty of Science, Medicine and Health - Papers: Part B. Retrieved from https://ro.uow.edu.au/ smhpapers1/1033 


\title{
Dasymaschalolactams A-E, Aristolactams from a Twig Extract of Dasymaschalon dasymaschalum
}

\author{
Abstract \\ Five new aristolactam alkaloids (1-5), dasymaschalolactams A-E, and the first isolation of \\ dasymaschalolactone (17) as a natural product, together with 19 known compounds (6-16 and 18-25) \\ were isolated from the twig extract of Dasymaschalon dasymaschalum. Their structures were elucidated \\ by spectroscopic methods as well as comparisons made from the literature. Compounds 20 and 21 \\ showed $\alpha$-glucosidase inhibitory activities with IC50 values of 4.5 and $24.7 \mu \mathrm{M}$, respectively.

\section{Publication Details} \\ Suthiphasilp, V., Maneerat, W., Andersen, R. J., Phukhatmuen, P., Pyne, S. G. \& Laphookhieo, S. (2019). \\ Dasymaschalolactams A-E, Aristolactams from a Twig Extract of Dasymaschalon dasymaschalum. \\ Journal of Natural Products, 82 (11), 3176-3180.

\section{Authors} \\ Virayu Suthiphasilp, Wisanu Maneerat, Raymond J. Andersen, Piyaporn Phukhatmuen, Stephen G. Pyne, \\ and Surat Laphookhieo
}




\section{Dasymaschalolactams A-E, Aristolactams from a}

\section{Twig Extract of Dasymaschalon dasymaschalum}

Virayu Suthiphasilp,,${ }^{\dagger, \ddagger}$ Wisanu Maneerat,,${ }^{\dagger} \ddagger$ Raymond J. Andersen, ${ }^{\S}$ Piyaporn Phukhatmuen, ${ }^{\dagger}$,

$\$$ Stephen G. Pyne ${ }^{\#}$ and Surat Laphookhieo ${ }^{*,+,}$

${ }^{\dagger}$ Center of Chemical Innovation for Sustainability (CIS) and ${ }^{\star}$ School of Science, Mae Fah Luang University, Chiang Rai 57100, Thailand

${ }^{\S}$ Department of Chemistry and Department of Earth, Ocean \& Atmospheric Sciences, University of British Columbia, 2036 Main Mall, Vancouver, BC, Canada V6T 1Z1

${ }^{\#}$ School of Chemistry and Molecular Biosciences, University of Wollongong, Wollongong, New South Wales 2522, Australia 
ABSTRACT: Five new aristolactam alkaloids (1-5), dasymaschalolactams A-E, and the first isolation of dasymaschalolactone (17) as a natural product, together with 19 known compounds (6-16, and 18-25) were isolated from the twig extract of Dasymaschalon dasymaschalum. Their structures were elucidated by spectroscopic methods as well as comparisons made from the literature. Compounds $\mathbf{2 0}$ and $\mathbf{2 1}$ showed $\alpha$-glucosidase inhibitory activities with $\mathrm{IC}_{50}$ values of 4.5 and $24.7 \mu \mathrm{M}$, respectively. 
Diabetes mellitus (DM) is a non-communicable disease that has become a major public health problem, affecting 1.6 million people worldwide in $20166^{1,2}$ This disease is characterized by hyperglycemia resulting from the inability to produce (type 1) or use insulin (type 2). ${ }^{3}$ The main risk factor leading to DM, especially type 2 , is food consumption ${ }^{4}$ leading to high glucose levels in the blood. $\alpha$-Glucosidases carry out the digestive breakdown of dietary polysaccharides, oligosaccharides, and disaccharides to glucose, ${ }^{5}$ a key step in the control of the amount of glucose in the blood. Drugs such as acarbose and miglitol used to decrease and control blood glucose levels are available on the market, but these can have undesired side effects and hence lead to poor compliance. ${ }^{6}$ Hence, the discovery of $\alpha$-glucosidase inhibitors from natural sources that can postpone the release of glucose from dietary carbohydrates and delay glucose absorption without or with less side effects is desirable.

Plants of the genus Dasymaschalon are small- to medium sized-trees belonging to the family Annonaceae. This genus consists of about 40 species worldwide that are distributed throughout tropical countries in Asia (particularly in Thailand and the Malaysian peninsular) and Africa. In Thailand, only 12 species are recognized. ${ }^{7}$ Previous phytochemical investigations on this genus have resulted in the isolation of various classes of compounds including flavonoids, ${ }^{8}$ triterpenoids, ${ }^{8}$ alkaloids, ${ }^{9,10}$ neolignans, ${ }^{11}$ benzyl benzoate derivatives, ${ }^{8,11}$ phenylpropanoids, ${ }^{12}$ acetogenins, ${ }^{13}$ and oxygenated cyclohexene derivatives. ${ }^{10,14}$ Some of these compounds have shown interesting biological properties including cytotoxicity, ${ }^{8-11,14}$ antimalarial, ${ }^{10}$ antiinflammatory ${ }^{14}$ and anti-HIV effects. ${ }^{12,14}$ Dasymaschalon dasymaschalum (Blume) I.M. Turner has many synonym names, including D. blumei Finet \& Gagnep., D. coelophloeum (Scheff.) Merr., and Desmos dasymaschalus (Blume) Saff. ${ }^{15}$ The powdered stems of this plant have been used as an antipyretic drug. ${ }^{9}$ The isolation of aristolactams, aporphine alkaloids, flavonoids, 
benzyl benzoate derivatives, and terpenoids have been reported from this plant. ${ }^{8,9}$ In a preliminary screening of the biological activities of Annonaceae plants, we found that the EtOAc extract of the twigs of $D$. dasymaschalum exhibited good $\alpha$-glucosidase inhibitory activity with $89 \%$ inhibition at $200 \mu \mathrm{g} / \mathrm{mL}$, which prompted an investigation of the chemical constituents from this extract. Herein, the isolation, structural elucidation, and $\alpha$-glucosidase inhibitory activities of the phytochemicals from this plant are described.

Twenty-five compounds, including 11 aristolactam alkaloids (1-11), one isoquinoline alkaloid (12), four flavonoids (13-16), a lactone (17), three benzyl benzoate derivatives (18, 19 and 25), two amides (20 and 21), two polyoxygenated cyclohexenes (22 and 23), and one benzene derivative (24) were isolated and characterized from the EtOAc extract of the twigs of D. dasymaschalum. All of the new compounds (1-5) were found to be aristolactam derivatives. Compound 17, an isobenzofuran-1(3H)-one derivative, was isolated for the first time from Nature. The known compounds were characterized as oldhamactam (6), ${ }^{16,17}$ velutinam (7), ${ }^{18}$ enterocarpam-III (8), ${ }^{19}$ griffithinam (9), ${ }^{20}$ goniopedalin (10), ${ }^{21}$ taliscanine $(\mathbf{1 1}),{ }^{21}$ duguevalline (12), ${ }^{22}$ desmethoxykanugin $(\mathbf{1 3}),{ }^{23}$ 7,8-dimethoxy-5-hydroxyflavone (14), ${ }^{24}$ alpinetin $(\mathbf{1 5}),{ }^{25} 8$ hydroxynaringenin-4'-methyl ether (16), ${ }^{26}$ 7-methoxyisobenzofuran-1(3H)-one (17), ${ }^{27}$ benzyl benzoate (18), ${ }^{28}$ 2-methoxybenzyl benzoate (19), ${ }^{28}$ paprazine $(\mathbf{2 0}),{ }^{29} \mathrm{~N}$-trans-feruloyltyramine $(\mathbf{2 1}),{ }^{30}(-)$-zeylenol $(\mathbf{2 2}),{ }^{31}(+)$-crotepoxide $(\mathbf{2 3}),{ }^{32}$ 4-hydroxybenzaldehyde $(\mathbf{2 4}),{ }^{33}$ and 2 phenylethyl benzoate $(\mathbf{2 5}),{ }^{34}$ by comparison of their spectroscopic and physical data with published values.

Dasymaschalolactam A (1) was isolated as a yellow viscous oil, and its molecular formula, $\mathrm{C}_{19} \mathrm{H}_{17} \mathrm{NO}_{5}$, was deduced from the NMR and the HRESITOFMS data, which displayed an ion at $m / z[\mathrm{M}+\mathrm{Na}]^{+} 362.1001$ (calcd for $\mathrm{C}_{19} \mathrm{H}_{17} \mathrm{NO}_{5} \mathrm{Na}, 362.1004$ ). The ${ }^{1} \mathrm{H}$ and ${ }^{13} \mathrm{C}$ NMR 
data of 1 (Tables 1 and 2) showed resonances for an $\mathrm{ABC}$ pattern of aromatic protons $\left[\delta_{\mathrm{H}} 8.86\right.$ (d, $J=8.5 \mathrm{~Hz}, \mathrm{H}-5), 7.51$ (t, $J=8.5 \mathrm{~Hz}, \mathrm{H}-6)$, and 7.17 (d, $J=8.5 \mathrm{~Hz}, \mathrm{H}-7)]$, one aromatic proton $\left[\delta_{\mathrm{H}} 7.73(\mathrm{~s}, \mathrm{H}-9)\right]$, four methoxy groups $\left[\delta_{\mathrm{H}} 4.51\left(\mathrm{~s}, \mathrm{CH}_{3} \mathrm{O}-2\right), 4.19\left(\mathrm{~s}, \mathrm{CH}_{3} \mathrm{O}-3\right), 4.00\left(\mathrm{~s}, \mathrm{CH}_{3} \mathrm{O}-\right.\right.$ 4), and $\left.4.07\left(\mathrm{~s}, \mathrm{CH}_{3} \mathrm{O}-8\right)\right]$, and one $\mathrm{NH}$ proton $\left[\delta_{\mathrm{H}} 9.86\right.$ (brs, $\left.\left.\mathrm{NH}\right)\right]$. The latter assignment was consistent with the IR spectrum, which displayed an absorption band at $3224 \mathrm{~cm}^{-1} .{ }^{35}$ The ${ }^{13} \mathrm{C}$ NMR spectrum of 1 displayed 19 resonances including those for $14 \mathrm{sp}^{2}$ carbons, one lactam carbonyl carbon, and four methoxy carbons. These NMR spectroscopic data were closely related to that of stigmalactam isolated from Fissistigma oldhamii. ${ }^{35}$ The major difference was the splitting pattern of the aromatic protons on the D ring. Stigmalactam showed an ABX pattern ${ }^{35}$ for H-5, H-7, and H-8 while compound 1 showed resonances for an ABC pattern for H-5, H-6, and $\mathrm{H}-7$. In addition, an additional methoxy proton resonance was also observed at $\delta_{\mathrm{H}} 4.07$ which was assigned to OMe- 8 on the basis of the HMBC correlations shown in Figure 1. The structure of compound $\mathbf{1}$ was determined as shown.

Dasymaschalolactam $B$ (2) gave the same molecular formula, $\mathrm{C}_{19} \mathrm{H}_{17} \mathrm{NO}_{5}$ [HRESITOFMS $\mathrm{m} / z 340.1186$ (calcd for $\mathrm{C}_{19} \mathrm{H}_{18} \mathrm{NO}_{5}, 340.1185$ )] as dasymaschalolactam A (1). The NMR data (Tables 1 and 2) as well as the HMBC correlations (Figure 1) were almost identical to those of $\mathbf{1}$, except that the ${ }^{1} \mathrm{H}$ NMR spectrum of $\mathbf{2}$ displayed an $\mathrm{ABC}$ pattern for H-6 $\left(\delta_{\mathrm{H}} 7.16, \mathrm{dd}, J=7.7,1.1 \mathrm{~Hz}\right), \mathrm{H}-7\left(\delta_{\mathrm{H}} 7.52, \mathrm{t}, J=7.7 \mathrm{~Hz}\right)$, and $\mathrm{H}-8\left(\delta_{\mathrm{H}} 7.49, \mathrm{dd}, J=7.7,1.1 \mathrm{~Hz}\right)$. The HMBC correlations shown in Figure 1 confirmed the structure of dasymaschalolactam B as shown.

The molecular formula of dasymaschalolactam C (3) $\left[\mathrm{C}_{18} \mathrm{H}_{15} \mathrm{NO}_{5}\right.$, HRESITOFMS $\mathrm{m} / \mathrm{z}$ $348.0849[\mathrm{M}+\mathrm{Na}]^{+}$(calcd for $\mathrm{C}_{18} \mathrm{H}_{15} \mathrm{NO}_{5} \mathrm{Na}, 348.0848$ ) $]$ indicated 3 to have one $\mathrm{CH}_{3}$ group less than 2. The NMR spectroscopic data of $\mathbf{3}$ (Tables 1 and 2) were almost identical to those of $\mathbf{2}$ 
except the methoxy group resonance $\left(\delta_{\mathrm{H}} 4.09\right)$ at C-5 of 2 was replaced by that for a hydroxy group $\left(\delta_{\mathrm{H}} 10.88\right)$ in $\mathbf{3}$. This was further confirmed by the HMBC correlations between the OH-5 $\left(\delta_{\mathrm{H}} 10.88\right)$ with $\mathrm{C}-4 \mathrm{~b}\left(\delta_{\mathrm{C}} 113.6\right), \mathrm{C}-5\left(\delta_{\mathrm{C}} 154.7\right)$, and C-6 $\left(\delta_{\mathrm{C}} 113.8\right)$. The structure of 3 was established as depicted.

Dasymaschalolactam D (4) gave a molecular formula of $\mathrm{C}_{18} \mathrm{H}_{16} \mathrm{NO}_{5}$, as indicated from its NMR and HRESITOFMS data $\left([\mathrm{M}+\mathrm{H}]^{+} m / z\right.$ 326.1031, calcd 326.1028). Analysis of the NMR spectroscopic data of $\mathbf{4}$ (Tables 1 and 2) showed that its structure is closely related to that of $\mathbf{3}$. The main difference found was that the methoxy group at C-4 of $\mathbf{3}$ was replaced by a hydroxy group in 4. This was further supported by the HMBC correlations shown in Figure 1. Hence, the structure of $\mathbf{4}$ was proposed as shown.

Dasymaschalolactam E (5) showed a molecular formula of $\mathrm{C}_{17} \mathrm{H}_{14} \mathrm{NO}_{4}$, which was deduced from its NMR and HRESITOFMS data $\left([\mathrm{M}+\mathrm{H}]^{+} \mathrm{m} / z\right.$ 296.0927, calcd 296.0923). Compound 5 was also demonstrated to be an aristolactam derivative, from its ${ }^{1} \mathrm{H}$ NMR spectroscopic data (Tables 1 and 2), which showed two singlet aromatic proton resonances at $\delta_{\mathrm{H}}$ $7.71(\mathrm{~s}, \mathrm{H}-2)$ and $7.14(\mathrm{~s}, \mathrm{H}-9)$, a set of $\mathrm{ABX}$ aromatic proton resonances at $\delta_{\mathrm{H}} 9.24(\mathrm{~d}, J=9.0$ Hz, H-5), 7.19 (dd, $J=9.0,2.8 \mathrm{~Hz}, \mathrm{H}-6)$, and 7.44 (d, $J=2.8 \mathrm{~Hz}, \mathrm{H}-8$ ), and two methoxy group resonances at $\delta_{\mathrm{H}} 3.96\left(\mathrm{~s}, \mathrm{CH}_{3} \mathrm{O}-7\right), 4.13\left(\mathrm{~s}, \mathrm{CH}_{3} \mathrm{O}-3\right)$. The observed $\mathrm{HMBC}$ correlations (Figure 1) confirmed the structure of $\mathbf{5}$ as shown.

Dasymaschalolactone, [7-methoxyisobenzofuran-1(3H)-one] (17), showed a protonated molecular ion at $\mathrm{m} / z$ 165.0553 $[\mathrm{M}+\mathrm{H}]^{+}$(calcd for 165.0552) in the HRESITOFMS, corresponding to the molecular formula $\mathrm{C}_{9} \mathrm{H}_{9} \mathrm{O}_{3}$. Compound 17 was a simple isobenzofuran1(3H)-one derivative that displayed ${ }^{1} \mathrm{H}$ NMR resonances for an $\mathrm{ABC}$ pattern of aromatic protons $\left[\delta_{\mathrm{H}} 7.16(\mathrm{dd}, J=7.5,0.8 \mathrm{~Hz}, \mathrm{H}-4), 7.72(\mathrm{dd}, J=8.2,7.5 \mathrm{~Hz}, \mathrm{H}-5)\right.$, and $\left.7.11(\mathrm{~d}, J=8.2 \mathrm{~Hz}, \mathrm{H}-6)\right]$, 
one oxymethylene group of a lactone ring $\left[\delta_{\mathrm{H}} 5.28(\mathrm{~s}, \mathrm{H}-3)\right]$, and one methoxy group [3.96 (s, $\left.\mathrm{CH}_{3} \mathrm{O}-5\right)$ ]. Compound 17 was one of the key products from the regiocontrolled rearrangement of isobenzofuran. ${ }^{27}$ This is the first report of the isolation of $\mathbf{1 7}$ from Nature.

Most of the isolated compounds (1-4, 7-8, 10-11, 13, 15-16, 18-21, and 24) were evaluated for their $\alpha$-glucosidase activity. Of the compounds listed, amides $\mathbf{2 0}$ and $\mathbf{2 1}$ exhibited good $\alpha$-glucosidase inhibitory activities with $\mathrm{IC}_{50}$ values at 4.5 and $24.7 \mu \mathrm{M}$, which were more active than the positive control, acarbose, $\left(\mathrm{IC}_{50}\right.$ value $\left.=73.7 \mu \mathrm{M}\right)$ while flavanone 16 showed a weaker $\alpha$-glucosidase inhibitory activity $\left(\mathrm{IC}_{50}\right.$ value $\left.=256.5 \mu \mathrm{M}\right)$ than that of acarbose.

\section{EXPERIMENTAL SECTION}

General Experimental Procedures. The general information on instruments and chromatographic materials was the same as in previous reports. ${ }^{36-40}$

Plant Material. The twigs of D. dasymaschalum were collected in May 2018 from Kuan Khao Wang Literary Botanic Garden, Chalung, Hat Yai District, Songkhla Province, Thailand. The plant was identified by one of the authors (S.L.) from a comparison with the authentic plant growing in Mae Fah Luang University. A voucher specimen (MFU-NPR0191) was deposited at the Natural Products Research Laboratory of Mae Fah Luang University.

Extraction and Isolation. The chopped and air-dried twigs of D. dasymaschalum (5.6 $\mathrm{kg}$ ) were extracted with EtOAc $(10 \mathrm{~L})$ at room temperature and concentrated under reduced pressure to provide an EtOAc extract (120.7 g). This crude extract was subjected to quick column chromatography (QCC) over silica gel (100\% hexanes to $100 \%$ EtOAc) to give eight fractions (Fr.D $1-$ Fr.D 8$)$. Fr.D 2 and Fr.D 3 were combined $(8.5 \mathrm{~g})$ and fractionated over Sephadex LH-20 (100\% MeOH) to give seven further fractions (Fr.D $2.3 \mathrm{~A}-$ Fr.D $\left.{ }_{2.3} \mathrm{G}\right)$. Compounds 22 (3.2 $\mathrm{mg})$ and $23(6.7 \mathrm{mg})$ were obtained from Fr. $\mathrm{D}_{2.3} \mathrm{D}(497.5 \mathrm{mg})$ by CC (3:2 acetone in hexanes). 
Purification of Fr.D ${ }_{2.3} \mathrm{E}(389 \mathrm{mg})$ by CC (1:3 EtOAc in hexanes) gave 11 (2.6 mg), together with 11 fractions (Fr.D ${ }_{2.3}$ EA - Fr.D $\left.{ }_{2.3} E U\right)$. Compound $2(7.2 \mathrm{mg})$ was obtained from Fr.D $2.3 \mathrm{EH}(10.5$ $\mathrm{mg})$ by CC (1:3 EtOAc in hexanes), whereas $1\left(3.1 \mathrm{mg}, t_{\mathrm{R}} 15.4 \mathrm{~min}\right), 3\left(2.5 \mathrm{mg}, t_{\mathrm{R}} 17.5 \mathrm{~min}\right)$, and $6\left(0.7 \mathrm{mg}, t_{\mathrm{R}} 20.1 \mathrm{~min}\right)$ were obtained from Fr. $\mathrm{D}_{2.3} \mathrm{D}(120 \mathrm{mg})$ by RP $\mathrm{C}_{18}$ HPLC (1.2:0.8 $\mathrm{CH}_{3} \mathrm{CN}$ in $\mathrm{H}_{2} \mathrm{O}, 2.0 \mathrm{~mL} / \mathrm{min}$ ). Fr.D $\mathrm{D}_{4}$ and Fr.D $\mathrm{D}_{5}$ were combined $(3.3 \mathrm{~g}$ ) and further separated by $\mathrm{CC}\left(1: 4 \mathrm{MeOH}\right.$ in $\left.\mathrm{CH}_{2} \mathrm{Cl}_{2}\right)$ to give six fractions (Fr.D $\left.{ }_{4.5} \mathrm{~A}-\mathrm{Fr} . \mathrm{D}_{4.5} \mathrm{~F}\right)$. Fr.D ${ }_{4.5} \mathrm{~B}(2.4 \mathrm{~g})$ was subjected to $\mathrm{CC}\left(1: 4 \mathrm{EtOAc}\right.$ in $\mathrm{CH}_{2} \mathrm{Cl}_{2}$ ) to yield six fractions (Fr.D ${ }_{4.5} \mathrm{BA}-\mathrm{Fr} . \mathrm{D}_{4.5} \mathrm{BF}$ ). Compound $19(3.7 \mathrm{mg})$ was isolated from Fr.D ${ }_{4.5} \mathrm{BB}(35.7 \mathrm{mg})$ by CC (2:4 EtOAc in hexanes). Purification of Fr.D ${ }_{4.5} \mathrm{BD}(98.7 \mathrm{mg})$ by CC (2:4 EtOAc in hexanes) yielded $18(5.7 \mathrm{mg})$. Purification of Fr.D ${ }_{4.5}$ C (40.2 mg) over Sephadex LH-20 (100\% MeOH) gave $14(4.2 \mathrm{mg})$ and $15(5.1 \mathrm{mg})$. Fractionation of Fr. $\mathrm{D}_{6}(2.34 \mathrm{~g})$ by Sephadex LH-20 (100\% MeOH) gave five fractions (Fr.D $\left.\mathrm{D}_{6} \mathrm{~A}-\mathrm{Fr} . \mathrm{D}_{6} \mathrm{E}\right)$. Compound $4(2.4 \mathrm{mg})$ was obtained from Fr.D ${ }_{6} \mathrm{C}(70.3 \mathrm{mg})$ by CC $(1: 4$ $\mathrm{MeOH}$ in $\mathrm{CH}_{2} \mathrm{Cl}_{2}$ ). Fr.D ${ }_{6} \mathrm{E}(257.4 \mathrm{mg})$ was subjected to RP $\mathrm{C}_{18} \mathrm{HPLC}\left(1.2: 0.8 \mathrm{CH}_{3} \mathrm{CN}\right.$ in $\mathrm{H}_{2} \mathrm{O}$, $2.0 \mathrm{~mL} / \mathrm{min})$ to give compound $7\left(1.3 \mathrm{mg}, t_{\mathrm{R}} 20.5 \mathrm{~min}\right)$. Separation of Fr. $\mathrm{D}_{6} \mathrm{D}(102.7 \mathrm{mg})$ over Sephadex LH-20 (100\% MeOH) yielded six subfractions (Fr.D 6 DA-Fr.D 6 DF). Compounds 20 $(10.5 \mathrm{mg})$ and $21(13.5 \mathrm{mg})$ were isolated from Fr.D ${ }_{6} \mathrm{DD}(51.6 \mathrm{mg})$ by $\mathrm{CC}(1: 4 \mathrm{MeOH}$ in $\left.\mathrm{CH}_{2} \mathrm{Cl}_{2}\right)$. Fr.D $\mathrm{D}_{6} \mathrm{DE}(30.5 \mathrm{mg})$ was subjected to Sephadex LH-20 CC $(100 \% \mathrm{MeOH})$ to yield compounds $12(0.5 \mathrm{mg})$ and $\mathbf{1 6}(3.5 \mathrm{mg})$. Compounds $5(3.0 \mathrm{mg}), \mathbf{8}(2.5 \mathrm{mg}), \mathbf{9}(2.0 \mathrm{mg})$, and $\mathbf{1 0}$ (2.1 mg) were obtained from Fr. $\mathrm{D}_{6} \mathrm{DF}(58.6 \mathrm{mg})$ by $\mathrm{CC}\left(1: 3 \mathrm{EtOAc}\right.$ in $\left.\mathrm{CH}_{2} \mathrm{Cl}_{2}\right)$ while $\mathbf{1 7}$ (1.5 $\mathrm{mg}), 24(2.5 \mathrm{mg})$, and $25(2.1 \mathrm{mg})$ were obtained from Fr. ${ }_{6} \mathrm{DB}(24.5 \mathrm{mg})$ by CC (1:4 MeOH in $\mathrm{CH}_{2} \mathrm{Cl}_{2}$.

Dasymaschalolactam A (1): yellow viscous oil; UV (MeOH) $\lambda_{\max }(\log \varepsilon) 358$ (2.76), 279 (3.58), 235 (3.52), $214(3.55) \mathrm{nm}$; IR (neat) $v_{\max } 3224,2998,1670,1448,1350,1287 \mathrm{~cm}^{-1} ;{ }^{1} \mathrm{H}$ 
and ${ }^{13} \mathrm{C}$ NMR, see Tables 1 and 2; HRESITOFMS $m / z$ 362.1001 $[\mathrm{M}+\mathrm{Na}]^{+}$(calcd for $\left.\mathrm{C}_{19} \mathrm{H}_{17} \mathrm{NO}_{5} \mathrm{Na}, 362.1004\right)$.

Dasymaschalolactam B (2): light yellow viscous oil; UV (MeOH) $\lambda_{\max }(\log \varepsilon) 399$ (2.66), 276 (3.39), 258 (3.44), 229 (3.29), 204 (3.38) nm; IR (neat) $v_{\max }$ 3241, 2939, 1688, 1378, 1083 $\mathrm{cm}^{-1} ;{ }^{1} \mathrm{H}$ and ${ }^{13} \mathrm{C}$ NMR, see Tables 1 and 2; HRESITOFMS $\mathrm{m} / z, 340.1186[\mathrm{M}+\mathrm{H}]^{+}$(calcd for $\left.\mathrm{C}_{19} \mathrm{H}_{18} \mathrm{NO}_{5}, 340.1185\right)$.

Dasymaschalolactam C (3): yellow viscous oil; UV (MeOH) $\lambda_{\max }(\log \varepsilon) 400$ (2.69), 257 (3.52), $233(3.37) \mathrm{nm}$; IR (neat) $v_{\max } 3203,2925,2858,1694,1468,1382 \mathrm{~cm}^{-1} ;{ }^{1} \mathrm{H}$ and ${ }^{13} \mathrm{C} \mathrm{NMR}$, see Tables 1 and 2; HRESITOFMS $m / z$ 348.0849 $[\mathrm{M}+\mathrm{Na}]^{+}$(calcd for $\mathrm{C}_{18} \mathrm{H}_{15} \mathrm{NO}_{5} \mathrm{Na}$, 348.0848).

Dasymaschalolactam D (4): brown viscous oil; UV (MeOH) $\lambda_{\max }(\log \varepsilon) 396$ (2.64), 276 (3.41), 258 (3.47), 233 (3.42), $204(3.32) \mathrm{nm}$; IR (neat) v $v_{\max } 3241,2935,1682,1383,1260,1076$ $\mathrm{cm}^{-1} ;{ }^{1} \mathrm{H}$ and ${ }^{13} \mathrm{C}$ NMR, see Tables 1 and 2; HRESITOFMS $\mathrm{m} / z$ 326.1031 $[\mathrm{M}+\mathrm{H}]^{+}$(calcd for $\left.\mathrm{C}_{18} \mathrm{H}_{16} \mathrm{NO}_{5}, 326.1028\right)$.

Dasymaschalolactam E (5): yellow viscous oil; UV (MeOH) $\lambda_{\max }(\log \varepsilon) 335$ (3.18), 284 (3.87), 234 (3.72), $215(3.75) \mathrm{nm}$; IR (neat) $v_{\max } 3224,2942,1678,1605,1376,1109,1035 \mathrm{~cm}^{-1}$; ${ }^{1} \mathrm{H}$ and ${ }^{13} \mathrm{C}$ NMR, see Tables 1 and 2; HRESITOFMS $m / z$ 296.0927 $[\mathrm{M}+\mathrm{H}]^{+}$(calcd for $\left.\mathrm{C}_{17} \mathrm{H}_{14} \mathrm{NO}_{4}, 296.0923\right)$.

Dasymaschalolactone (17): colorless viscous oil; UV (MeOH) $\lambda_{\max }(\log \varepsilon) 295$ (3.17), 237 (3.64), 208 (4.35) nm; IR (neat) $v_{\max }$ 2950, 2901, 1750, 1604, $1180 \mathrm{~cm}^{-1} ;{ }^{1} \mathrm{H}$ NMR (600 MHz, acetone- $\left.d_{6}\right) \delta_{\mathrm{H}} 7.72(1 \mathrm{H}, \mathrm{dd}, J=8.2,7.5 \mathrm{~Hz}, \mathrm{H}-5), 7.16(1 \mathrm{H}, \mathrm{dd}, J=7.5,0.8 \mathrm{~Hz}, \mathrm{H}-4)$, $7.11(1 \mathrm{H}, \mathrm{d}, \mathrm{J}=8.2, \mathrm{H}-6), 5.28(1 \mathrm{H}, \mathrm{s}, \mathrm{H}-3), 3.96(3 \mathrm{H}, \mathrm{s}, \mathrm{OMe}-7) ;{ }^{13} \mathrm{C}$ NMR $(150 \mathrm{MHz}$, acetone$\left.d_{6}\right) \delta_{\mathrm{C}} 169.5(\mathrm{C}-1), 158.6(\mathrm{C}-7), 150.1(\mathrm{C}-3 \mathrm{a}), 135.6(\mathrm{C}-5), 113.5,(\mathrm{C}-4), 112.9(\mathrm{C}-7 \mathrm{a}), 110.2(\mathrm{C}-$ 
6), 67.8 (C-3), 59.2 (OMe-7); HRESITOFMS m/z $165.0553[\mathrm{M}+\mathrm{H}]^{+}$(calcd for $\mathrm{C}_{9} \mathrm{H}_{9} \mathrm{O}_{3}$, 165.0552).

$\alpha$-Glucosidase inhibitory Assay. A colorimetric $\alpha$-glucosidase (Sigma, St. Louis, MO, USA, cat. No. G5003) assay was performed using the previously reported method. ${ }^{40}$ Acarbose was used as a positive control with an $\mathrm{IC}_{50}$ value of $73.7 \mu \mathrm{M}$.

\section{ASSOCIATED CONTENT}

\section{Supporting Information}

The Supporting Information is available free of charge on the ACS Publications website at

DOI:

HRESITOFMS, 1D and 2D NMR spectra of new compounds 1-5 and 17 (PDF).

\section{AUTHOR INFORMATION}

\section{Corresponding Author}

*Tel: +66-5391-6238. Fax: +66-5391-6776. E-mail: surat.lap@mfu.ac.th (S. Laphookhieo).

\section{ORCID}

Raymond J. Andersen: 0000-0002-7607-8213

Stephen G. Pyne: 0000-0003-0462-0277

Surat Laphookhieo: 0000-0002-4757-2781

\section{Notes}

The authors declare no competing financial interest.

\section{ACKNOWLEDGMENTS}

This work was supported by Thailand Research Fund (BRG5980012, DBG6280007 and PHD/0133/2559) and Mae Fah Luang University. Mae Fah Luang University and University of British Columbia are also acknowledged for laboratory facilities. 


\section{REFERENCES}

(1) Syed, A. T. Int. J. Health Sci. 2007, 1, 5-8.

(2) World Health Organization, The Top 10 Causes of Death (Fact sheet), 2018. https://www.who.int/news-room/fact-sheets/detail/the-top-10-causes-of-death (accessed 10 May 2019).

(3) American Diabetes Association Diabetes Care. 2013, 36 (Suppl. 1), 67-74.

(4) Hamilton, M. T.; Hamilton, D. G.; Zderic, T. W. Med. Sport. Sci. 2014, 60, 11-26.

(5) Kumar, S.; Narwal, S.; Kumar, V.; Prakash, O. Pharmacogn. Rev. 2011, 5, 19-29.

(6) Kooti, W.; Farokhipour, M.; Asadzadeh, Z.; Ashtary-Larky, D.; Asadi-Samani, M. Electron. Physician 2016, 8, 832-1842.

(7) Wang, J.; Chalermglin, J.; Saunders, R. M. K. System. Bot. 2009, 34, 252-265.

(8) Prawat, U.; Chairerk, O.; Lenthas, R.; Salae, A. W.; Tuntiwachwuttikul, P. Phytochem. Lett. 2013, 6, 286-290.

(9) Chanakul, W.; Tuchinda, P.; Anantachoke, N.; Pohmakotr, M.; Piyachaturawat, P.; Jariyawat, S.; Suksen, K.; Jaipetch, T.; Nuntasaen, N.; Reutrakul, V. Fitoterapia 2011, 82, 964-968.

(10) Jaidee A.; Promchai, T.; Trisuwan, K.; Laphookhieo, S.; Rattanajak, R.; Kamchonwongpaisan, S.; Pyne, S. G.; Ritthiwigrom, T. Nat. Prod. Commun. 2015, 10, $1175-1177$.

(11) Hongthing, S.; Kuhakarn, C.; Jaipetch, T.; Piyachaturawat, P.; Jariyawat, S.; Suksan, S.; Limthongkul, J.; Nuntasaen, N.; Reutrakul, V. Nat. Prod. Commun. 2016, 11, 809-813.

(12) Yu, Z. X.; Niu, Z. G.; Li, X. B.; Zheng, C. J.; Song, X. M.; Chen, G. Y.; Song, X. P.; Han, C. R.; Wu, S. X. Fitoterapia 2017, 118, 27-31. 
(13) Sinz, A.; Matusch, R.; Kampchen, T.; Fiedler, W. Helv. Chim. Acta 1998, 81, $1608-1615$.

(14) Hongthong, S.; Kuhakarn, C.; Jaipetch, T.; Prabpai, S.; Kongsaeree, P.; Piyachaturawat, P.; Jariyawat, S.; Suksan, K.; Limthingkul, J.; Panthong, A.; Nuntasaen, N.; Reutrakul, V. Fitoterapia 2015, 106, 158-166.

(15) Plant List, version 1. http://www.theplantlist.org/tpl1.1/record/kew-2604143, 2019. (Accessed May 18, 2019).

(16) Li, S. H. Zhongguo Yaoxue Zazhi 2014, 49, 1503-1505.

(17) Zhou, X. L.; Wu, J. H.; Bai, J.; Hu, X. L.; Li, E. Z.; Shi, N.; Pei, Y. H. Chin. J. Nat. Med. 2013, 11, 81-83.

(18) Yang, X. N.; Jin, Y. S.; Zhu, P.; Chen, H. S. Chem. Nat. Compd. 2010, 46, 324-326.

(19) Nayyatip, S.; Thaichana, P.; Buayairaksa, M.; Tuntiwechapikul, W.; Meepowpan, P.; Nuntasaen, N.; Pompimon, W. Int. J. Mol. Sci. 2012, 13, 5010-5018.

(20) Zhang, Y. J.; Kong, M.; Chen, R. Y.; Yu, D. Q. J. Nat. Prod. 1996, 62, 1050-1052.

(21) Talapatra, S. K.; Basu, D.; Chattopadhyay, P.; Talapatra, B. Phytochemistry 1988, 27, 903-906.

(22) Perez, E.; Saez, J.; Blair, S.; Franck, X.; Figadere, B. Lett. Org. Chem. 2004, 1, $102-104$.

(23) Das, B.; Kumar, A.; Chakravarty, K.; Masuda, K.; Suzuki, H.; Ageta, H. Phytochemistry 1994, 37, 1363-1366.

(24) Escarria, S. R.; Torrenegra, R. D.; Angarita, B. Phytochemistry 1977, 16, 1618.

(25) Xiao, X.; Si, X.; Tong, X.; Li, G. Sep. Purif. Technol. 2011, 81, 265-269.

(26) Braca, A.; Bilia, A. R.; Mendez, J.; Morelli, I. Phytochemistry 1999, 51, 1125-1138. 
(27) Egan, B. A.; Paradowski, M.; Thomas, L. H.; Marquez, R. Org. Lett. 2011, 13, 2086-2089.

(28) Kodpinid, M.; Sadavongvivad, C.; Thebtaranonth, C.; Thebtaranonth, Y. Phytochemistry 1984, 23, 199-200.

(29) Rahman, A. U.; Bhatti, M. K.; Akhtar, F.; Choudhary, M. I. Phytochemistry 1992, 31, 2869-2872.

(30) Sarker, S. D.; Bartholomew, B.; Nash, R. J. Fitoterapia 2000, 71, 328-330.

(31) Jolad, S. D.; Hoffmann, J. J.; Schram, K. H.; Cole, J. R. J. Org. Chem. 1981, 46, 4267-4272.

(32) Kupchan, S. M.; Hemingway, R. J.; Smith, R. M. J. Org. Chem. 1969, 34, 3898-3902.

(33) Zhou, C. C.; Liu, C. T.; Huang, X. X.; Wu, J.; Li, L. Z.; Li, D. N.; Song, S. J. Zhongguо Yaоwи Hиахие Zazhi 2013, 23, 213-217.

(34) Formisano, C.; Rigano, D.; Senatore, F.; Raimondo, M.; Maggio, A.; Bruno, M. Nat. Prod. Commun. 2012, 7, 1379-1382.

(35) Chia, Y. C.; Chang, F. R.; Teng, C. M.; Wu, Y. C. J. Nat. Prod. 2000, 63, $1160-1163$.

(36) Raksat, A.; Maneerat, W.; Andersen, R. J.; Pyne, S. G.; Laphookhieo, S. J. Nat. Prod. 2018, 81, 1835-1840.

(37) Jaidee, W.; Andersen, R. J.; Chavez, M. A. G.; Wang, Y. A.; Patrick, B. O.; Pyne, S. G.; Muanprasat, C.; Borwornpinyo, S.; Laphookhieo, S. J. Nat. Prod. 2019, 82, 283-292.

(38) Sriyatep, T.; Siridachakorn, I.; Maneerat, W.; Pansanit, A.; Ritthiwigrom, T.; Andersen, R. J.; Laphookhieo, S. J. Nat. Prod. 2015, 78, 265-271. 
(39) Sriyatep, T.; Andersen, R. J.; Patrick, O. B.; Pyne, S. G.; Muanprasat, C.; Seemakhuan, S.; Borwornpinyo, S.; Laphookhieo, S. J. Nat. Prod. 2017, 80, 1658-1667.

(40) Meesakul, P.; Richardson, S.; Pyne, S. G.; Laphookhieo, S. J. Nat. Prod. 2019, 82, $741-747$. 


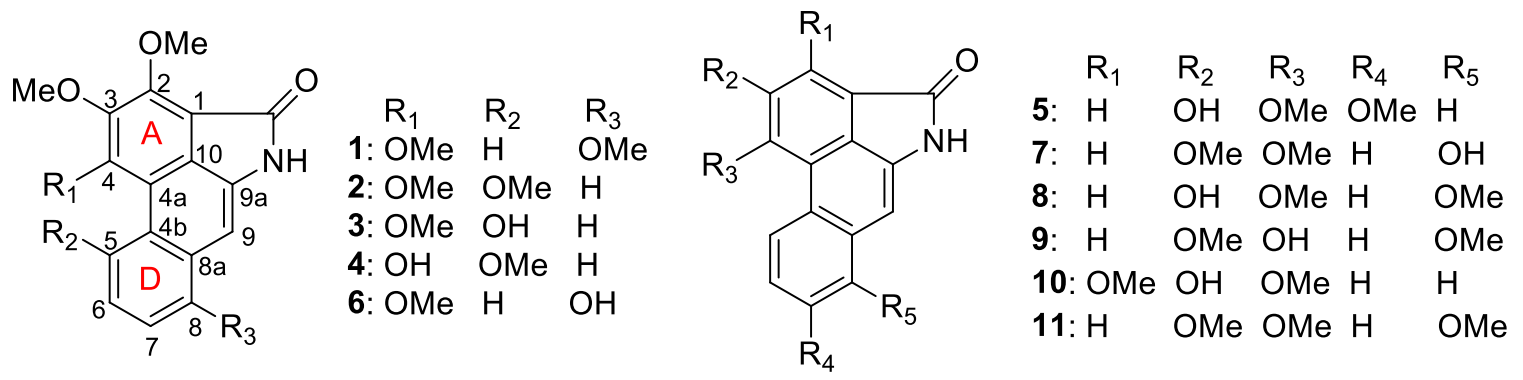<smiles>COc1cc(OC)c2c(c1)C(=O)C1=NC=Cc3c(OC)c4c(c1c3-2)OCO4</smiles><smiles>[R]c1ccc(-c2oc3c([R])c([R])cc([R3])c3c(=O)c2[R4])cc1[R6]</smiles><smiles>COc1ccc([C@@H]2CC(=O)c3c(O)cc(O)c(O)c3O2)cc1</smiles>

$\begin{array}{llllll}\mathrm{R}_{1} & \mathrm{R}_{2} & \mathrm{R}_{3} & \mathrm{R}_{4} & \mathrm{R}_{5} & \mathrm{R}_{6}\end{array}$ 13: $\mathrm{H} \quad \mathrm{OMe} \mathrm{H} \quad \mathrm{OMe} \quad \mathrm{OCH}_{2} \mathrm{O}$ 14: $\mathrm{OMe} \mathrm{OMe} \mathrm{OH} \mathrm{H} \quad \mathrm{H} \quad \mathrm{H}$ 15: $\mathrm{H} \quad \mathrm{OH} \quad \mathrm{OMeH} \quad \mathrm{H} \quad \mathrm{H}$

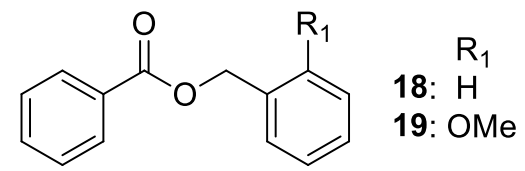<smiles>[R]c1cc(/C=C/C(=O)NCCc2ccc(O)cc2)ccc1O</smiles>
20: $\mathrm{H}$

21: $\mathrm{OMe}$<smiles>[Z4]c1ccc(C(=O)O)cc1</smiles><smiles>O=C(OCCc1ccccc1)c1ccccc1</smiles>

Chart 1. Compounds isolated from the twig extracts of D. dasymaschalum. 

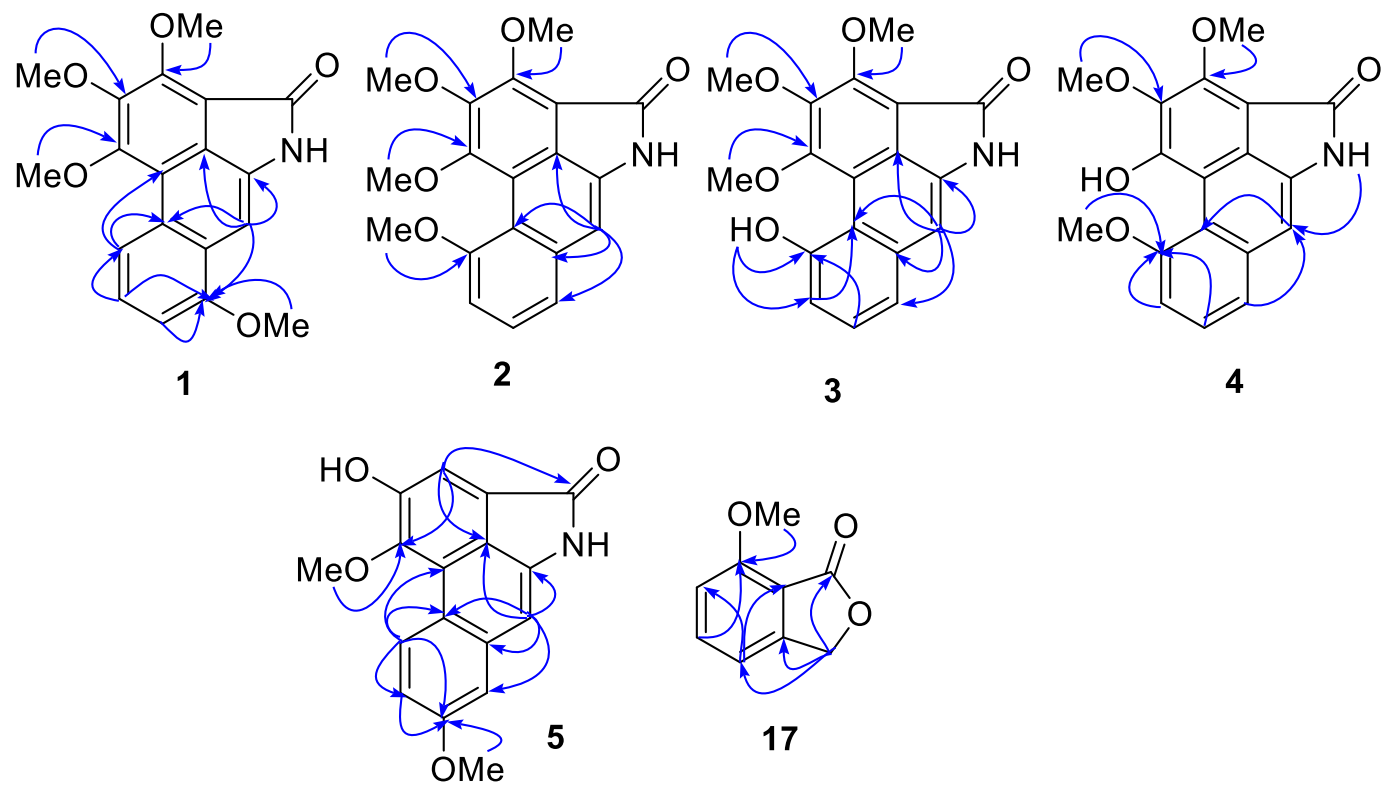

Figure 1. Key HMBC correlations for compounds 1-5 and 17 
Table 1. ${ }^{1} \mathrm{H}$ NMR (600 MHz) Spectroscopic Data of Compounds 1-5 in Acetone- $d_{6}(\delta$ in ppm, $J$ in $\mathrm{Hz}$ )

\begin{tabular}{llllll} 
position & $\mathbf{1}$ & $\mathbf{2}$ & $\mathbf{3}$ & $\mathbf{4}$ & $\mathbf{5}$ \\
2 & & & & $7.71, \mathrm{~s}$ \\
5 & $8.86, \mathrm{~d}(8.5)$ & & & & $9.24, \mathrm{~d}(9.0)$ \\
6 & $7.51, \mathrm{t}(8.5)$ & $7.16, \mathrm{dd}(7.7,1.1)$ & $7.06, \mathrm{dd}(7.7,1.4)$ & $7.13, \mathrm{dd}(7.6,1.1)$ & $7.19, \mathrm{dd}(9.0,2.8)$ \\
7 & $7.17, \mathrm{~d}(8.5)$ & $7.52, \mathrm{t}(7.7)$ & $7.49, \mathrm{t}(7.7)$ & $7.52, \mathrm{t}(7.6)$ & \\
8 & & $7.49, \mathrm{dd}(7.7,1.1)$ & $7.45, \mathrm{dd}(7.7,1.4)$ & $7.49, \mathrm{dd}(7.6,1.1)$ & $7.44, \mathrm{~d}(2.8)$ \\
$8 \mathrm{a}$ & & & & \\
9 & $7.73, \mathrm{~s}$ & $7.19, \mathrm{~s}$ & $7.29, \mathrm{~s}$ & $7.20, \mathrm{~s}$ & $7.14, \mathrm{~s}$ \\
OMe-2 & $4.51, \mathrm{~s}$ & $4.44, \mathrm{~s}$ & $4.14, \mathrm{~s}$ & $3.90, \mathrm{~s}$ & $4.13, \mathrm{~s}$ \\
OMe-3 & $4.19, \mathrm{~s}$ & $3.95, \mathrm{~s}$ & $4.47, \mathrm{~s}$ & $3.98, \mathrm{~s}$ & \\
OMe-4 & $4.00, \mathrm{~s}$ & $3.93, \mathrm{~s}$ & $4.08, \mathrm{~s}$ & & \\
OH-5 & & & $10.88, \mathrm{~s}$ & & \\
OMe-5 & & $4.09, \mathrm{~s}$ & & $4.08, \mathrm{~s}$ & \\
OMe-7 & & & & & \\
OMe-8 & $4.07, \mathrm{~s}$ & & & $9.62, \mathrm{~s}$ & \\
$\mathrm{NH}$ & $9.86, \mathrm{br} \mathrm{s}$ & $9.78, \mathrm{~s}$ & $9.96, \mathrm{~s}$ & \\
\hline
\end{tabular}

Table 2. ${ }^{13} \mathrm{C}$ NMR (150 MHz) Spectroscopic Data Compounds 1-5 in Acetone- $d_{6}(\delta$ in ppm)

\begin{tabular}{llllll} 
position & $\mathbf{1}$ & $\mathbf{2}$ & $\mathbf{3}$ & $\mathbf{4}$ & $\mathbf{5}$ \\
1 & 108.9 & 111.4 & 111.6 & 104.5 & 106.5 \\
2 & 153.6 & 153.1 & 151.5 & 156.5 & 106.1 \\
3 & 156.4 & 156.7 & 152.7 & 143.4 & 147.8 \\
4 & 146.0 & 146.5 & 146.4 & 149.6 & 147.6 \\
$4 \mathrm{a}$ & 115.5 & 114.0 & 115.1 & 112.8 & 120.3 \\
$4 \mathrm{~b}$ & 126.7 & 115.6 & 113.6 & 116.1 & 114.5 \\
5 & 118.4 & 157.2 & 154.7 & 156.9 & 128.8 \\
6 & 124.9 & 106.6 & 113.8 & 106.6 & 113.4 \\
7 & 105.9 & 126.8 & 127.9 & 120.6 & 157.9 \\
8 & 155.1 & 120.3 & 120.2 & 126.2 & 109.9 \\
$8 \mathrm{a}$ & 123.8 & 135.4 & 135.9 & 135.3 & 136.9 \\
9 & 98.2 & 104.5 & 106.3 & 104.9 & 103.6 \\
$9 \mathrm{a}$ & 133.5 & 133.0 & 133.7 & 125.9 & 116.4 \\
10 & 125.1 & 126.8 & 125.5 & 128.3 & 123.8 \\
$\mathrm{C}=$ O & 165.7 & & & 166.9 & 168.2 \\
OMe-2 & 61.7 & 61.6 & 63.3 & 59.7 & \\
OMe-3 & 59.8 & 59.9 & 61.9 & 60.1 & 56.3 \\
OMe-4 & 60.5 & 60.3 & 60.9 & & \\
OMe-5 & & 54.8 & & 54.8 & \\
OMe-6 & & & & & 54.3 \\
OMe-7 & 54.8 & & & & \\
\hline
\end{tabular}




\section{Table of Content}

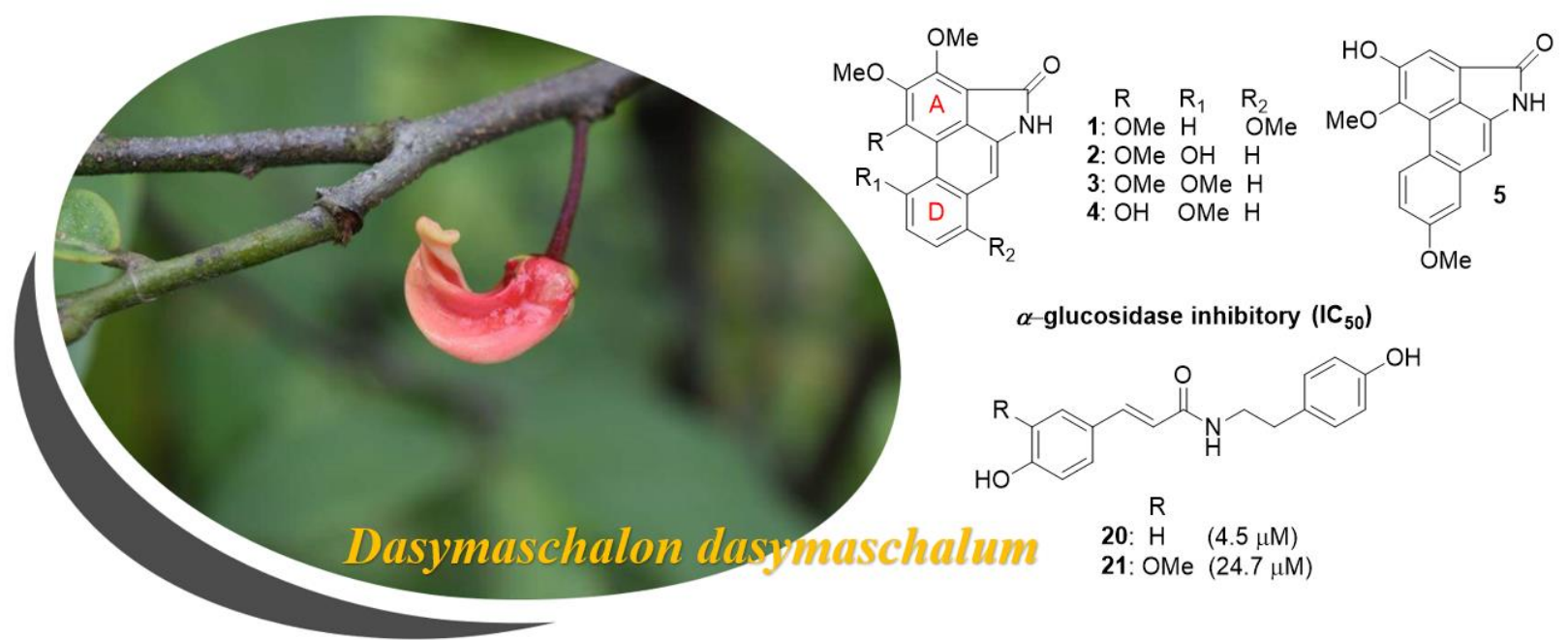

